v. 8 - n. 2- agosto-dezembro/2011 - ISSN: 1806-5023

\title{
FEDERALISMO Y GOBERNABILIDAD: UN BREVE ANÁLISIS EN ARGENTINA Y BRASIL
}

\section{Michelle Vieira Fernández ${ }^{1}$}

Hay un movimiento general, académico incluso e institucional, para acomodar en la sociedad los distintos intereses [...]

Gilberto Gil, Ministro de Cultura de Brasil.

El País, 04/03/2008.

\section{INTRODUCCIÓN}

En un mundo compuesto por pluralismo de culturas y diversidad de intereses es fundamental el intento de consensuar las diferentes opciones y voluntades. En el campo político, la tendencia debe ir en la misma dirección, se busca un modelo de democracia consensual como promotora de una sociedad más igualitaria en la medida que este tipo de democracia intenta promover el consenso en detrimento de una dictadura de la mayoría (LIJPHART, 2007). En este escenario global marcado por la diversidad ubicamos al Federalismo como un modelo de democracia que recoge a lo que podríamos llamar de consenso.

Actualmente $40 \%$ de la población vive en países de estructura federal o parafederal, distribuidos en veinticinco federaciones en todos los continentes (GRIFFITHS, 2002). Es decir, aunque solamente

\footnotetext{
${ }^{1}$ Universidad de Salamanca - España. E-mail: mfernandez@usal.es
} 
una pequeña parcela de países sean federales casi la mitad de la población mundial vive bajo las reglas del juego del federalismo, mejor dicho, están sometidas a instituciones federales, lo que señala el federalismo como tema de agenda importante para los estudios políticos.

En los países latinoamericanos, cuyas sociedades son plurales y heterogéneas, el federalismo tiene mucho que aportar, principalmente, por las diferencias en el desarrollo económico de las distintas regiones, provincias, entidades federativas y municipios. Sin embargo, sólo algunos países son federales, entre ellos Brasil y Argentina.

A partir de la relación establecida entre pluralismo, consenso y federalismo surge una serie de preguntas para la implementación de buenos gobiernos, sobre todo en países en vías de desarrollo, como es el caso de los países latinoamericanos, es decir: ¿es posible asociar pluralismo y efectividad de los gobiernos?; ¿es posible establecer el consenso a la hora de elaborar las leyes que son necesarias para poner en prácticas los planes de gobierno?

Para estas preguntas, los sistemas federales que aquí serán discutidos ya dieron la respuesta: el federalismo y la gobernabilidad no son variables opuestas, es decir, es posible conjugar en un mismo escenario el pluralismo político y la capacidad de actuación de los gobierno. La nueva pregunta que surge, y que va a guiar este trabajo, es si la variación en el "grado" de federalismo² puede influir en la gobernabilidad del sistema político.

En este trabajo intentaré llevar a cabo la relación entre el grado de federalismo y la gobernabilidad, en concreto trataré de una de las dimensiones de este concepto, la efectividad política, que va a ser

\footnotetext{
${ }^{2}$ De acuerdo con Vallès (2007, p. 183), bajo el rotulo de Federación o estado federal están incluidas configuraciones muy dispares y practicas muy diferentes, lo que lleva a concluir que no existe un único modelo federal. A partir de estas observaciones es que se puede plantear una variación en el grado de federalismo.
} 
medido por la capacidad del ejecutivo de aprobar sus leyes en el parlamento.

Al realizar un análisis comparativo entre Argentina y Brasil trataré de ubicar estos países en una escala de federalismo a partir de algunas variables propuestas por Lijphart (2007) y Stepan (2001) en sus trabajos. El objetivo principal es saber si la ubicación de los países en esta escala tiene relación con la gobernabilidad en los términos presentados aquí.

\section{1 ¿QUE ENTENDEMOS POR GOBERNABILIDAD?}

La referencia a la gobernabilidad se hace constante en los estudios de los sistemas políticos mundiales y en especial en los estudios políticos de las realidades de América Latina. En los países menos desarrollados, como son los países latinoamericanos, la gobernabilidad ha adquirido una notable rasgo socioeconómico, es decir, los factores políticos de la misma se contemplan, de manera general, en su capacidad de producir resultados económicos que aseguren el crecimiento y la superación de la marginación social y de la pobreza (Alcántara, 1995, p. 24; Munk, 2003). Para entender la capacidad de resolver los problemas socioeconómicos en este tipo de escenario político, es decir, para analizar la capacidad de mantener el equilibrio entre los inputs y los outputs en el sistema político, resulta fundamental estudiar la manera en la que el gobierno se ocupa y desarrolla sus funciones. Así, gobernabilidad alude a la capacidad política y administrativa de gobernar.

La definición de gobernabilidad varía en una escala pequeña de un autor para otro. De acuerdo con Bourricaud (apud ALCÁNTARA, 1995), gobernabilidad es un conjunto de condiciones que aseguran el ejercicio del poder en una sociedad sin excesivo riesgo de que esta llegara a desarticularse violentamente. Yendo más allá de concepto de Bourricaud, Flisfisch (apud ALCÁNTARA, 1995) afirma que para la consecución de la gobernabilidad no basta la pura operación de las 
reglas del juego democrático, lo que exige un orden como resultado de la operación de esas reglas. Ese orden genera, entonces, una situación de equilibrio social generalizado y relativamente estable. Además, para Flisfisch, es necesario observar en la definición de gobernabilidad las dimensiones de efectividad y eficacia de las decisiones adoptadas (ALCÁNTARA, 1995, p 39). Así, el concepto de gobernabilidad adoptado para este trabajo es el que toma porgobernabilidad la situación en que concurre un conjunto de condiciones favorables para la acción de gobierno.

A partir de lo expuesto anteriormente, se puede asociar la gobernabilidad con la estabilidad gubernamental, es decir, se puede afirmar que la existencia de condiciones favorables para la acción del gobierno proporciona un gobierno estable. La estabilidad gubernamental puede ser entendida por la unión de tres factores: la duración del gobierno, que mide la estabilidad de los gobiernos por el tiempo de ocupación del poder; el grado de control del ejecutivo, que indica si el gobierno cuenta o no con la mayoría legislativa; y el nivel de violencia política, que es incapacidad del sistema político para institucionalizar el conflicto (ALCÁNTARA, 1995, p. 28). A partir de estos factores surge una secuencia en cadena: gobernabilidad, estabilidad y democracia de calidad.

La literatura que trabaja el tema de gobernabilidad trae al campo teórico la constatación de que la gobernabilidad es un fenómeno pluridimensional (ALCÁNTARA, 1995; ARBÓS Y GINER, 1996; HEREDIA VARGAS, 2002). A partir de esta constatación, plantean el análisis de la gobernabilidad a partir de dos dimensiones, es decir, legitimidad y eficacia ${ }^{3}$.

Hay autores que ponen el acento en la eficacia para analizar la gobernabilidad, mientras que otros se detienen más en las cuestiones de legitimidad. Tomando como punto inicial el planteamiento de los

\footnotetext{
${ }^{3}$ Para una discusión más detenida sobre las dos dimensiones de la gobernabilidad, legitimidad y eficacia, sobre todo sobre la eficacia que es la dimensión que no se trabaja en este articulo ver HEREDIA VARGAS (2002).
} 
autores que ponen el acento en la eficacia y poniendo de manifiesto las limitaciones de espacio y tiempo de este trabajo, será usado para medir el grado de gobernabilidad en las sociedades estudiadas aquí una fracción del dilema legitimidad-eficacia, es decir, la relación entre el ejecutivo y el legislativo a la hora de aprobar los proyectos de ley que provienen de las manos presidenciales. Por lo tanto, intentaré analizar la eficacia del gobierno, es decir, la capacidad de poner en practicas sus políticas, a través de su capacidad de aprobación de leyes en sus Cámaras de Diputados.

En las democracias del Tercer Mundo, las relaciones entre ejecutivo y legislativo son condición política especial, entre otras, que realzan el significado de las variables políticas para entender las recurrentes crisis de gobernabilidad. Esta relación conflictiva entre ejecutivo y legislativo es motivo para que algunos autores (LIJPHART, 1992; LINZ, 1988) manifiesten una "preferencia" por los sistemas parlamentarios, donde el ejecutivo tiene implícitamente la mayoría en el congreso, en detrimento de los sistemas presidenciales, donde una mayoría para la aprobación de las leyes debe ser construida, mucha veces a duras penas. Es por estos motivos que se enfatiza, en general, que los gobiernos de coalición, mucha veces necesarios en los sistemas presidenciales y predominantes en el escenario político de muchos países, se encuentran sometidos a una buena cantidad de disfunciones resultantes de su propia naturaleza, que llegan a afectar seriamente a su rendimiento ya que comprometen la puesta en marcha de las políticas del gobierno (ALCÁNTARA, 1995, p. 61).

A partir de esta perspectiva, entonces, se analiza a seguir el "éxito legislativo" como uno de los factores de la eficacia política de los gobiernos. Más precisamente, se considera la relación entre el número de iniciativas que presenta el Ejecutivo y el Legislativo en una legislatura con el número de iniciativas provenientes de los mismos 
actores que son aprobadas para medir este éxito legislativo ${ }^{4}$ en Argentina y Brasil (GARCíA, 2006, p. 75). En este estudio, el foco se mantiene en el éxito legislativo del ejecutivo, aunque se considere el éxito legislativo en general a modo comparativo.

\section{CONSIDERACIONES SOBRE EL FEDERALISMO}

Etimológicamente, el término federalismo proviene del latín foedus, que quiere decir "contrato", "tratado" o "convenio". De esta definición lingüística se desdoblan muchas otras, sin embargo todas ellas se centran en un mismo punto: el acto de compartir el poder a través de diversos niveles de organización del aparato estatal.

Las federaciones son estados compuestos que constan de distintas unidades políticas con sus respectivos gobiernos y de un gobierno central. Cada uno de estos niveles de gobierno posee una serie de poderes o competencias que han sido delegados por el pueblo a través de la aprobación de una constitución común. Bajo normas federales, las organizaciones políticas combinan el autogobierno de las unidades constituyentes (provincias, estados, länder, etc.) con el gobierno compartido, por este motivo la esencia del principio normativo del federalismo se basa simultáneamente en la unidad nacional del Estado y su autonomía política interna (NOVESÀ, 2005).

El federalismo es clasificado por Vallès (2007) como un Estado compuesto. Para él, en los Estados compuestos el poder político es repartido entre instituciones que controlan ámbitos territoriales diferentes, es decir, hay un acuerdo político de partida para compartir poder entre niveles de gobierno sin que haya la imposición de un centro político único que se imponga a los demás actores

\footnotetext{
${ }^{4}$ La autora tiene conciencia de que el éxito legislativo no es suficiente para medir la gobernabilidad en un sistema político, sin embargo, como fue explicitado a lo largo de este apartado, este es un factor que compone la compleja ecuación de la gobernabilidad y que debe ser tomado en consideración. Por lo tanto, ese trabajo es simplemente un primer paso en la escalera del análisis de correlación entre federalismo y gobernabilidad y abre una agenda importante de investigación para estos temas en sistemas políticos como los de Argentina y Brasil.
} 
institucionales en todos los campos de intervención. Así, podemos entender el federalismo como principio organizador del poder y de la descentralización.

Vallès (2007) apunta cinco principales características del federalismo: distribución constitucional de competencias con algunas estrictas que corresponden a la federación; reparto de la capacidad tributaria; existencia de instituciones federales en cuya composición y actividad intervienen las entidades federadas; existencia de una constitución propia en cada una de las entidades federadas; y existencia de un tribunal central o federal para arbitrar en los conflictos de la federación con los estados o en los conflictos de los estados entre sí.

Sin embargo, Watts (1996) afirma que la característica esencial de las federaciones es que se componen de dos - o más - niveles de gobierno y que funcionan dentro de una estructura constitucional en la que se combina el gobierno compartido sobre algunas cuestiones específicas, a través de instituciones comunes, y el autogobierno regional para otros asuntos concretos, mediante los gobiernos de las unidades territoriales constitutivas.

Según Stepan (2001), las federaciones tienden a restringir los poderes del centro en la medida que el centralismo dificulta la consolidación democrática ya que puede centralizar el poder de agenda política, limitar la representación y participación de otros grupos o sectores, y limitaría el flujo de recursos económicos de áreas prioritarias de política pública.

La regionalización es un proceso encaminado a institucionalizar un pluralismo político de base territorial; supone la distribución, el reparto y la difusión del poder del Estado por el territorio nacional. Por este motivo, en los países con regímenes federales, la entidad que recibe las tareas estatales es la región o la entidad federativa; siendo así, el Estado centralista o unitario desaparece y los estados miembros pueden influir a través de los mecanismos institucionales 
en las decisiones del poder central. De acuerdo con Vallès (2007), los componentes de esta red de multiplicidad de poderes que configura la estructura federal ni siempre mantienen entre sí una clara configuración jerárquica. El autor afirma además que esta pluralidad de intervenciones plantea problemas para hacer efectiva la responsabilidad democrática.

Así, la esencia del federalismo está en la creación de instituciones y procesos que permitan alcanzar una unidad política que acomode y aumente la diversidad durante la solución de los problemas de la vida diaria. Se entiende entonces que el federalismo puede conciliar intereses, valores, preferencias diversas que son inherentes a la sociedad. El objeto del federalismo de reconciliar unidad con diversidad a pesar de parecer utópico es una realidad inevitable en el escenario político-social plural en que vivimos.

\subsection{NUESTRA ESCALA DE FEDERALISMO.}

Para crear una escala del federalismo, este trabajo vuelve a las bases teóricas de los estudios de Lijphart (1992; 2007) agregando algunas observaciones hechas por Stepan (2001). Arend Lijphart (2007), en "Modelos de Democracia", construye un mapa bidimensional de la democracia y sitúa en un extremo el mayoritarismo y en el otro el consenso. En la realización de este estudio el autor ubica en un modelo dicotómico treinta y seis democracias.

En el análisis son tomadas instituciones y normas democráticas importantes para deducir diez diferencias de los principios mayoritarios y consensuales y son agrupadas estas diez variables en dos dimensiones. Es decir, por un lado está la dimensión ejecutivospartidos y por otro la dimensión federal-unitaria. Para el análisis propuesto en el presente trabajo vamos a utilizar para medir el federalismo en Argentina y Brasil las variables de la segunda dimensión creada por Arend Lijphart, exceptuando la variable División 
del poder, ya que serán analizados aquí solamente países federales. Es decir, las variables de Lijphart incorporadas aquí son: Parlamentos y Congresos; Constituciones; Constitucionalidad; y Bancos Centrales. Lijphart (2007, p. 179) afirma que

[...] una legislatura bicameral con una camera federal fuerte para representar las regiones constituyentes, una constitución difícil de enmendar, un tribunal supremo o un tribunal constitucional especial que puede proteger la constitución mediante su poder de revisión judicial y un banco centra independiente [...] son 'normas del federalismo.

De acuerdo con él, estos factores son los que garantizan que la división básica del poder sea preservada, es decir, estos elementos, más que componentes, son elementos que garantizan el federalismo.

La variable Parlamentos y Congresos se refiere a la distribución, concentración frente a la división, del poder en el legislativo. En cuanto a esta distribución del poder en los parlamentos, la clasificación trabajada por Lijphart (2007) varia en grados del unicameralismo al bicameralismo, pero para este trabajo nos detuvimos en variaciones del bicameralismo ya que en sistemas federales los parlamentos son bicamerales. Esta clasificación se centra en cuánto poder posee cada una y qué representa cada cámara, es decir, mide el grado de congruencia y de simetría. "Por congruente [...] se entiende a los sistemas bicamerales en los que ambas cámaras se eligen con la misma base de representación y la misma forma de elección, dando lugar a composiciones político partidarias similares - congruentes - en ambas cámaras" (REYNOSO, 2009: 107). Ya cuando se hace referencia a la simetría, tratan-se de cámaras con el mismo grado de poder, o con competencias no lo suficientemente distintas como para considerar que una es más poderosa que la otra; en contraposición, cuando una de las cámaras es dotada con menos poderes que la otra, estamos tratando de una cámara asimétrica. Así, a partir de lo presentado, los países pueden 
ser clasificados como: Bicameralismo débil (Cámaras asimétricas y Congruentes); Bicameralismo semifuerte I (Cámaras asimétricas e incongruentes); Bicameralismo semifuerte II (Cámaras asimétricas y congruentes); y Bicameralismo fuerte (Cámaras simétricas e incongruentes).

La variable Constituciones hace referencia a la facilidad o dificultad de introducir enmiendas a la Constitución. La clasificación propuesta a partir de la rigidez o facilidad de enmendar la constitución está relacionada con las exigencias para la aprobación en una votación de cambio constitucional en la Asamblea. Por lo tanto, las posibles clasificaciones generadas son: Mayorías simples; Mayorías entre simples y de dos tercios; Mayorías de dos tercios o equivalentes; y Mayorías superiores a dos tercios.

La variable Constitucionalidad trata de la presencia o ausencia de revisión judicial para resolver conflictos entre la constitución y la ley común. La clasificación de los países de acuerdo con la fuerza de la revisión judicial se basa, en primer lugar, en la distinción entre la presencia y la ausencia de revisión judicial y, en segundo lugar, en tres grados de activismo en la afirmación de este poder por los tribunales. Así, la clasificación a partir de los criterios supra citados se presenta de la siguiente manera: Sin revisión judicial; Revisión judicial débil; Revisión judicial semifuerte; y Revisión judicial fuerte.

La variable Bancos Centrales trata de la dependencia o independencia del Banco Central con relación al gobierno. Para determinar si los Bancos Centrales de los países estudiados son fuertes se utiliza el índice de Cukierman, Webb y Neyapti LIJPHART (2007, p. 219-220). Para la construcción de este índice los autores analizan dieciséis variables relacionadas con la independencia legal de los bancos centrales. Su índice global es un promedio de las dieciséis variables - que varían entre 0 y 1 . Hay cuatro agrupaciones de variables en la construcción de este índice: el nombramiento y mandato del gobernador; la formulación de políticas; los objetivos del 
Banco Central; y los límites sobre los préstamos. Para facilitar la clasificación de los Bancos Centrales, se determinar para el presente análisis que se considere la siguiente clasificación: 0 para Banco Central Dependiente; 0,1 a 0,4 para Banco Central con Independencia Débil; 0,41 a 0,7 para Banco Central con Independencia Fuerte; y 0,71 a 1 para Banco Central con Independencia Plena 5 .

Cuando se mira hacia Stepan (2001), se encuentra una agenda de investigación abierta y orientada por el estudio de cuatro variables estrechamente relacionadas con el diseño constitucional y con los partidos políticos. La primera de ellas, y la que vamos incorporar al presente análisis, es El grado de representación en la cámara territorial, en donde la sobrerrepresentación de los estados pequeños genera alto potencial para la cámara alta limitar las mayorías. Esta relación tiene fuerte efecto sobre las políticas públicas. Esta variable es medida por el coeficiente de Gini. Para efectos de análisis, la escala considerada para este trabajo es la siguiente: los índices de Gini hasta 0,10 como Demos Enaibling Débil; de 0,11 hasta 0,34 Demos Enaibling Fuerte; de 0,36 hasta 0,51 como Demos Constraining Débil y a partir de 0,52 como Demos Constraining Fuerte. Estos umbrales fueron fijados a partir de las constataciones hechas por Stepan (2001) en su trabajo.

Las otras tres variables propuestas por Stepan son: el ámbito de políticas públicas bajo la competencia de la cámara territorial; el grado en que la capacidad de legislar inscrita en la constitución depende de súper mayorías o de las subunidades; y el grado en que el sistema de partidos cuenta con sistemas de orientación e incentivos repartidos en todo el territorio nacional. Estas tres variables se quedan fuera de este análisis por distintos motivos. Las dos primeras no son incluidas en la construcción de una escala de

\footnotetext{
${ }^{5}$ Clasificación elaborada a partir de la observación de la Tabla con los Índices de Independencia del Banco Central utilizados por Lijphart (2007, p. 221) y de las características de los diversos Banco Centrales analizados por este autor tomando como punto e partida la tabla antes referida
} 
federalismo porque están estrechamente relacionadas con la variable dependiente de este análisis, es decir, con la gobernabilidad en los términos planteados aquí. Ya la última variable, el grado en que el sistema de partidos cuenta con sistemas de orientación e incentivas repartidos en todo el territorio nacional, por la dificultad de medirla en los países propuestos para este análisis ${ }^{6}$.

A partir de este recogido por los trabajos de Lijphart (1992; 2007) y Stepan (2001) quedan fijadas las variables que serán utilizadas aquí para analizar el sistema político federal de Argentina y Brasil'. Así, las cinco variables utilizadas aquí para la creación de una nueva escala de federalismo son: Parlamentos y Congresos; Constituciones; Constitucionalidad; Bancos Centrales; Grado de Sobrerrepresentación en las Cámaras Territoriales.

\section{LA GOBERNABILIDAD Y EL FEDERALISMO EN ARGENTINA $Y$ BRASIL}

\subsection{LA GOBERNABILIDAD EN ARGENTINA Y BRASIL}

Como ya fue explicado anteriormente, la dimensión de la gobernabilidad que será analizada a seguir es la que concierne a la efectividad estudiada en este texto por intermedio de la variable "éxito legislativo", con hincapié en las actividades legislativas del poder ejecutivo.

Fue observado la capacidad de aprobación de las leyes del poder ejecutivo en el legislativo federal, es decir, la relación entre la cantidad de leyes presentadas por el presidente y la cantidad de

\footnotetext{
${ }^{6}$ Esta variable podría ser medida a través de Índice de Distribución Territorial (IDT) de los Partidos Políticos. Por las dimensiones de este articulo fue imposible elaborar tal índice para Brasil y Argentina. Simón Pachano en sus trabajos sobre división territorial del poder, elaboró el mismo índice para los países andinos. Para el IDT en la Región Andina ver PACHANO (2004). Por lo tanto, queda por desarrollarse esta propuesta de investigación.

${ }^{7}$ La autora tiene conciencia de que el modelo de análisis propuesto por Lijphart no es el único modelo que hay en la literatura. Sin embargo, este es un modelo de análisis ampliamente aceptado entre los estudiosos del tema, por eso fue el modelo adoptado para este estudio.
} 
leyes aprobadas dentro de un mismo intervalo de tiempo. El periodo analizado aquí son los años 2002 y 2003. Estos dos años, en ambos países, fue un periodo de término de mandato presidencial y comienzo de un nuevo mandato.

Argentina y Brasil poseen sistemas políticos presidenciales y multipartidistas. En estos sistemas políticos es muy difícil que el presidente llegue a asumir el poder ya con una mayoría formada en el Congreso. Por lo tanto, al empezar sus actividades, el presidente electo comienza a desarrollar una ardua tarea: la de construir una mayoría en el Congreso que le posibilite aprobar sus leyes.

En el periodo analizado aquí, la base de apoyo aproximada que tenían los presidentes de ambos países varia entre $45 \%$ y $60 \%$, lo que les posibilitaba mantener un elevado nivel de éxito legislativo, como señalan los dados descritos a seguir.

Tabla I: Éxito Legislativo en Brasil y Argentina

\begin{tabular}{|c|c|c|c|c|}
\hline $\begin{array}{c}\text { PAÍS/ NUMERO } \\
\text { DE } \\
\text { PROYECTOS }\end{array}$ & $\begin{array}{c}\text { EJECUTIVO } \\
\text { PRESENTADO }\end{array}$ & $\begin{array}{c}\text { EJECUTIVO } \\
\text { APROBADO }\end{array}$ & $\begin{array}{c}\text { TOTAL } \\
\text { PRESENTADO }\end{array}$ & $\begin{array}{c}\text { TOTAL } \\
\text { APROBADO }\end{array}$ \\
\hline BRASIL & 108 & 41 & 4338 & 132 \\
\hline ARGENTINA & 172 & 113 & 5449 & 313 \\
\hline
\end{tabular}

Fuente: Elaboración propia a partir de datos de las Cámaras de Diputados de Argentina y Brasil.

De acuerdo con los datos presentados, tanto en Brasil como en Argentina la cantidad de proyectos presentados por el legislativo es muy superior a la cantidad presentada por el ejecutivo, es decir, en Brasil, en el periodo analizado, fueron presentados un total de 4338 proyectos y entre estos solo 108 son originalmente del ejecutivo. En Argentina, de los 5449 proyectos presentados entre 2002 y 2003 son provenientes del poder ejecutivo solamente 172 . 
A partir de la observación de los datos es posible afirmar que los regímenes presidencialistas de Argentina y Brasil logran mantener buenos niveles de éxito legislativo aunque tengan que convivir con la pluralidad dentro del Congreso y con la tarea de construir mayorías y de formar consensos. Sin embargo, aunque sea pequeña, existe diferencia entre el número de propuestas presentas por el ejecutivo y que fueron aprobadas en los dos países. La pregunta que sigue, entonces, es si las diferencias institucionales que componen el federalismo en Argentina y Brasil pueden influir en sus niveles de gobernabilidad expresa a través del éxito legislativo, es decir, en la aprobación de leyes originarias del ejecutivo. Es lo que veremos más adelante en este texto.

\subsection{EL FEDERALISMO EN ARGENTINA Y BRASIL.}

Como ya fue mencionado anteriormente, en América Latina, con sus sociedades plurales y heterogéneas, las diferencias en el desarrollo económico de las distintas regiones, provincias, entidades federativas y municipios hacen que el federalismo sea un mecanismo institucional adecuado. Sin embargo, solo cuatro países en esta región son formalmente federales, Venezuela, México, Brasil y Argentina, y de estos tres son federales de hecho, México, Brasil y Argentina.

Para el presente análisis serán abordados los casos federales de Brasil y Argentina. Estos dos países fueron elegidos por poseer antecedentes históricos semejantes. Los dos son sistemas políticos que pasaron por dictaduras en la misma época y tienen, por lo tanto, una historia democrática reciente.

\subsubsection{El Federalismo en Argentina}

El federalismo argentino se creó como una forma de organización política y territorial para agregar y luego acomodar en su seno a un buen número de unidades autónomas que presentaban 
importantes disparidades entre sí (NOVESÀ, 2005). A seguir serán demostrados algunos aspectos que caracterizan el sistema político argentino y sus propiedades federales.

\subsubsection{Parlamentos y Congresos}

El Congreso Argentino es formado por dos Cámaras, el Senado de la Nación y la Cámara de Diputados. Ambas Cámaras son elegidas por el pueblo a través de voto directo.

La Cámara de Diputados está formada por 257 parlamentarios, mientras que el Senado de la Nación está compuesto por 72 senadores. Los diputados son elegidos de manera proporcional al número de electores de los distritos electorales. La magnitud de los distritos para la elección de diputados varía de provincia en provincia. Los diputados se renuevan por mitades a cada dos años y los mandatos tienen duración de cuatro años.

Los senadores son distribuidos de la siguiente manera: tres por cada provincia, dos elegidos por el partido que obtenga más votos y uno por el partido que le sigue en número de votos. Se renueva por tercios y tienen mandatos de seis años. Cada distrito posee tres senadores.

Así, el bicameralismo argentino es caracterizado como simétrico, las dos Cámaras tienen poderes constitucionales iguales, e incongruente, las dos Cámaras tienen composiciones diferentes. Por lo tanto, y de acuerdo con la topología de Lijphart (2007), se puede clasificar Argentina como un Bicameralismo fuerte.

\subsubsection{Constituciones}

La Constitución argentina de 1994 parece ser atípica por su concreción, considerando que está se encuentra en el grupo de Constituciones de última generación (MELO, 2008). Argentina posee una constitución sucinta que fue promulgada en 1994. Desde 
entonces, la constitución nunca recibió enmiendas. De acuerdo con Melo (2008), se observa una correlación negativa entre la extensión de la Constitución y el grado de dificultad para realizar enmiendas. Además, el autor observa que las constituciones que más enmiendas recibe son las que son más flexibles, es decir, las que son más fáciles de ser enmendadas. Esto indica, entonces, que la constitución de Argentina se caracteriza por ser una constitución rígida, exigiendo para ser enmendada Mayoría de dos tercios ${ }^{8}$.

\subsubsection{Constitucionalidad}

Hay revisión de la constitucionalidad para los casos en que sea solicitado9 ${ }^{9}$ Esta revisión judicial en Argentina es de forma descentralizada, es decir, existe más de un órgano que realiza la revisión. Aquí la revisión judicial puede ser ejecutada por la Corte Suprema o por los Tribunales inferiores de la Nación. De esta manera, la Constitucionalidad en el sistema federal argentino puede ser clasificada como Constitucionalidad Semifuerte.

\subsubsection{Bancos Centrales}

El significado del termino independencia tiene aparecido en literatura como la capacidad del Banco Central en no ceder frente a las fuerzas políticas para monetizar grandes déficits presupuestario. Es decir, hay la necesidad de independencia institucional en relación a los poderes Ejecutivo y Legislativo (FERREIRA, 2006).

\footnotetext{
${ }^{8}$ Artículo 30 de la Constitución Argentina: “La Constitución puede reformarse en el todo o en cualquiera de sus partes. La necesidad de reforma debe ser declarada por el Congreso con el voto de dos terceras partes, al menos, de sus miembros; pero no se efectuará sino por una Convención convocada al efecto".

${ }^{9}$ Artículo 116 de la Constitución de Argentina: “Corresponde a la Corte Suprema y a los tribunales inferiores de la Nación, el conocimiento y decisión de todas las causas que versen sobre puntos regidos por la Constitución, y por las leyes de la Nación, con la reserva hecha en el inciso 12 del Artículo 75; y por los tratados con las naciones extranjeras; de las causas concernientes a embajadores, ministros públicos y cónsules extranjeros; de las causas de almirantazgo y jurisdicción marítima; de los asuntos en que la Nación sea parte; de las causas que se susciten entre dos o más provincias; entre una provincia y los vecinos de otra; entre los vecinos de diferentes provincias; y entre una provincia o sus vecinos, contra un Estado o ciudadano extranjero".
} 
De acuerdo con el Índice creado por Cukierman, Webb y Neyapti (1992), y con todas las características que este índice lleva en consideración, se puede decir que Argentina tiene un alto grado de independencia del Banco Central, en el periodo llevado en consideración ${ }^{10}$, con un Índice de Independencia del Banco Central de 0,77 . Así de acuerdo con la clasificación descrita anteriormente, consideramos el Banco Central Argentino como un Banco Central con Independencia Plena.

\subsubsection{Grado de Representación en la Cámara Territorial}

Las unidades federales están capacitadas para participar e influenciar en el proceso de toma de decisiones a escala federal por medio de algunas instituciones como la segunda cámara, es decir, el Senado de la Nación.

Con el principal argumento de garantizar cierta igualdad de condiciones entre todas las provincias, se establece en argentina un Senado en el que cada una de las provincias contará con igual representación $\mathrm{y}$, por tanto, no se tienen en cuenta criterios poblacionales, de extensión del territorio u otros a la hora de atribuir un número de senadores por provincia (NOVESÀ, 2005).

Así, pues, la atribución de un número igual de senadores a las provincias implica la sobrerrepresentación de las menos pobladas y, por consiguiente, la infrarrepresentación de las más pobladas. Por consiguiente, la provincia de Tierra del Fuego, con 115.000 habitantes, le corresponde el mismo número de senadores que la provincia de Buenos Aires con 11 millones de habitantes. Los cuatro distritos que representan el 64 por ciento de la población (las provincias de Buenos Aires, Santa Fe, Córdoba y la Ciudad de Buenos Aires) sólo controlan el 17 por ciento del Senado. Tras la presentación

${ }^{10}$ Datos de 2000. Índice disponible en Hicks (2003). 
de estos datos queda claro la falta de proporcionalidad en cuanto a la representación de la periferia.

Argentina, por consiguiente, se sitúa dentro del grupo de países con mayor grado de sobrerrepresentación de las entidades subestatales menos pobladas. De acuerdo con la clasificación de Stepan (2001) Argentina es Demos Constraining Fuerte, es decir, tiene capacidad de restringir la mayoría y presenta un Índice de Gini de 0,61 - el más alto entre los países medidos por Stepan.

\subsubsection{El Federalismo en Brasil}

En 1985, Brasil deja un Periodo de Dictadura Militar y vuelve a un sistema político democrático. Pero, la inauguración efectiva de este nuevo periodo democrático brasileño se dio en 1988 con la promulgación de una nueva carta Constitucional Brasileña que instituyó un sistema político basado en una República Federativa presidencialista, con bicameralismo simétrico e incongruente, representación proporcional de listas abiertas y separación de poderes.

En medio de procesos de centralización en periodos autoritarios y descentralización en períodos democráticos, el concepto de federación se confunde con el concepto de democracia en si mismo. Así, medidas centralizadoras se identifican como autoritarias, mientras que la descentralización se considera democrática (REGIS, 2009).

\subsubsection{Parlamentos y Congresos}

El Congreso brasileño esta formado por dos Cámaras simétricas e incongruentes. Las Cámaras simétricas son aquellas que poseen poder constitucional iguales o ligeramente desiguales y de legitimidad democrática, es decir, sistemas donde la segunda Cámara, en nuestro caso el Senado Federal, no son elegidos de forma indirecta y tampoco son nombrados. Además, las dos Cámaras son 
incongruentes cuando la segunda, es decir, el Senado, es elegida siguiendo distinto método de la elección en la primera Cámara (LIJPHART, 2007, p. 194-195).

La Cámara de Diputados y el Senado Federal son elegidos de forma directa por los ciudadanos. Los diputados tienen mandatos de 4 años y son electos de manera integral, es decir, la Cámara se renueva toda de una sola vez. Ya los senadores tienen mandatos de 8 años y son electos de manera fraccionada, es decir, un tercio en una elección y dos tercios en la siguiente.

La Cámara de Diputados posee 513 diputados que son electos de manera proporcional a cada distrito electoral ${ }^{11}$. El Senado Federal, donde están representados los estados de la federación, está constituido por 81 senadores, tres senadores por cada uno de los veintiséis estados y tres para el Distrito Federal, electos por el sistema mayoritario.

De acuerdo con lo descrito anteriormente, y con las características tipológicas definidas por Lijphart y usadas en este análisis, se puede decir que el sistema federal brasileño es caracterizado por un Bicameralismo fuerte.

\subsubsection{Constituciones}

La Constitución brasileña es una de las más largas de América Latina (MELO, 2008). Esta es una constitución que además de ser muy larga y detallada es de susceptible a la aceptación de enmiendas.

La elevada tasa de enmiendas encontrada en el caso brasileño es consistente con la baja rigidez constitucional en el país. Hay pocas barreras para la proposición de enmiendas constitucionales y el proceso legislativo de enmiendas presenta pocos obstáculos debido al quórum calificado relativamente bajo ( $3 / 5$ o $60 \%$ de los miembros del

11 En Brasil los distritos electorales coinciden con las unidades de la federación, así que tenemos 27 distritos electorales. 
Congreso en las dos Cámaras, en contraste con la exigencia de 2/3 más común en la región y también adoptada en la Constitución brasileña de 1946), aunque sean necesarias dos votaciones en las dos Cámaras ${ }^{12}$.

Una singularidad brasileña es la inexistencia de quórum diferenciado según los artículos a ser enmendados. El quórum para artículos de mayor relevancia institucional -como los que se refieren a los tres poderes- es semejante a los que reglamentan disposiciones comunes.

De esta manera, se puede clasificar la constitución brasileña, de acuerdo con la topología de Lijphart preestablecida, como una constitución poco rígida que establece como requisito para ser enmendada Mayorías entre ordinarias y de dos tercios.

\subsubsection{Constitucionalidad}

12 Articulo 60 de la Constitución de Brasil: “La Constitución podrá ser enmendada mediante propuesta:

I. - de un tercio, por lo menos, de los miembros de la Cámara de Diputados o del Senado Federal;

II. - del Presidente de la República;

III. - - de más de la mitad de las Asambleas Legislativas de las unidades de la Federación, manifestándose, cada una de ellas, por la mayoría relativa de sus miembros.

- $\quad \S 1.0$ La Constitución no podrá ser enmendada en la vigencia de intervención federal, de estado de defensa o de estado de sitio.

- $\quad \S 2$. ․ La propuesta será discutida y votada en cada Cámara del Congreso Nacional, en dos turnos, considerándose aprobada si se consigue, en ambos, tres quintos de los votos de los respectivos miembros.

- $\quad$ 3.․ La enmienda a la Constitución será promulgada por las Mesas de la Cámara de Diputados y del Senado Federal, con el respectivo número de orden.

0

$\S 4$. N No será objeto de deliberación la propuesta de enmienda tendiente a abolir:
I. - la forma federativa de Estado;
II. - el voto directo, secreto, universal y periódico;
III. - - la separación de los Poderes;
IV. $\quad-$ los derechos de garantias individuales.

- $\S 5.0$ La materia constante de propuesta de enmienda rechazada o habida por perjudicada no puede ser objeto de nueva propuesta en la misma sesión legislativa. (Traducción propia del original en Portugués). 
En Brasil, el poder delegado por la constitución para el judiciario es el de otorgar constitucionalidad de las leyes creadas por el legislativo ${ }^{13}$. Posee una Revisión Judicial Fuerte ya que el papel de revisar la constitución esta centrado en el Supremo Tribunal Federal.

\subsubsection{Bancos Centrales}

${ }^{13}$ Articulo 102 de la Constitución de Brasil: “Compete al Supremo Tribunal Federal vigilar de la Constitución:

I - Procesar y juzgar, originariamente:

$\alpha$. La acción directa de inconstitucionalidad de ley o acto normativo federal o estatal y la acción declaratoria de constitucionalidad de ley o acto normativo federal;

* Redacción dada por la Enmienda Constitucional 03/93 - D.O.U. 18.03.93.

$\quad \beta . \quad$ En las infracciones penales comunes, el Presidente de la República, el Vice-Presidente, los miembros del Congreso Nacional, sus propios Ministros y el Procurador-General de la República;

$\chi$. En las infracciones penales comunes y en los crímenes de responsabilidad, los Ministros de Estado y los Comandantes de la Marina, del Ejército y de la Aeronáutica, excepto el dispuesto en el art. 52, I, los miembros de los Tribunales Superiores, los del Tribunal de Cuentas de la Unión y los jefes de misión diplomática de carácter permanente;

* Redacción dada por la Enmienda Constitucional 23/99 - D.O.U. 03.09.99.

$\delta$. $\quad$ El habeas-corpus, siendo paciente cualquiera de las personas referidas en las "alineas" anteriores; el mandado de seguridad y el hábeasdata en contra actos del Presidente de la República, de las Mesas de la Cámara de Diputados y del Senado Federal, del Tribunal de Cuentas de la Unión, del Procurador-General de la República y del propio Supremo Tribunal Federal;

ع. El litigio entre Estado extranjero o organismo internacional y la Unión, el Estado, el Distrito Federal o el Territorio;

$\phi . \quad$ Las causas y los conflictos entre la Unión y los Estados, la Unión y el Distrito Federal, o entre unos y otros, inclusive las respectivas entidades de la administración indirecta;

$\gamma . \quad$ La extradición solicitada por Estado extranjero;

$\eta$. La homologación de las sentencias extranjera y la concesión del "exequátur" a las cartas de petición, que pueden ser conferidas por el reglamento interno a su Presidente;

$(\ldots)$

III - Juzgar, mediante recurso extraordinario, las causas decididas en única o última instancia, cuando la decisión recurrida: 
La independencia del Banco Central reside en la capacidad de buscar prioritariamente el objetivo de estabilidad de precios, aunque para esto los objetivos conflictivos de corto plazo, como por ejemplo el empleo pleno, sean sacrificados (HOLANDA ET AL., 2002 ).

De acuerdo con el Índice creado por Cukierman, Webb y Neyapti (1992), y con todas las características que este índice lleva en consideración, es correcto afirmar que Brasil tiene un bajo grado de independencia del Banco Central, en el periodo llevado en consideración ${ }^{14}$, con un Índice de Independencia del Banco Central de 0,20 . Así de acuerdo con la clasificación descrita anteriormente, consideramos el Banco Central Brasileño como un Banco Central con Independencia Débil.

\subsubsection{Grado de Representación en la Cámara Territorial}

El Senado Federal de Brasil esta formado por 81 senadores, tres de cada unidad de la federación, como ya fue dicho anteriormente. La igualdad de peso entre los senadores de las diferentes unidades federativas hace con que hay un alto grado de sobrerrepresentación en la segunda cámara brasileña. Por ejemplo, si se considera la unidad de la federación más pequeña, Roraima, y la más grande, São Paulo, se percibe esta constatación de manera muy clara. Un voto,

a. Contrariar dispositivo de esta Constitución;

b. Declarar la inconstitucionalidad de tratado o ley federal;

c. Juzgar válida ley o acto del gobierno local impugnado contra esta Constitución.

\section{$[\ldots]$}

$\S 20$ - Las decisiones definitivas de mérito, proferidas por el Supremo Tribunal Federal, en las acciones declaratorias de constitucionalidad de ley $o$ acto normativo federal, producirán eficacia contra todos y efecto vinculante, relativamente a los demás órganos del Poder Judiciario e al Poder Ejecutivo.

* Redacción por la Enmienda Constitucional 03/93 - D.O.U. 18.03.93.

(Traducción propia del original en Portugués).

${ }^{14}$ Datos de 2000. Índice disponible en Hicks (2003). 
para el Senado, en Roraima tiene 144 veces más valor que un voto en São Paulo.

De igual modo que Argentina, Brasil se sitúa dentro del grupo de países con mayor grado de sobrerrepresentación de las entidades subestatales menos pobladas. Como demuestra Stepan (2001) Brasil es Demos Constraining Fuerte, es decir, tiene capacidad de restringir la mayoría, y presenta un Índice de Gini de 0,52 - el segundo más alto, superado solamente por Argentina, entre los países medidos por Stepan.

\section{LA RELACIÓN ENTRE FEDERALISMO $Y$ NIVELES DE GOBERNABILIDAD EN LOS CASOS ANALIZADOS}

En sus trabajos sobre Federalismo Stepan (2001) atribuye gran importancia al tema de la eficacia, pues, para él, en términos institucionales la exigencia de mayorías absolutas o calificadas, puede disminuir el riesgo de bloqueos dados por las votaciones de las minorías. Pero a la vez, la puesta en prácticas de principios federalistas en el parlamento puede dar más oportunidad a otros actores de participar en el control político.

De esta manera lo esperado es que en sistemas federativos la capacidad del ejecutivo de hacer con que sus leyes sean aprobadas en el Congreso sea baja. Sin embargo, como ya fue señalado anteriormente, esto no es lo que ocurre en los sistemas federales de América Latina analizados en este texto. Como se constata en el gráfico abajo, la capacidad de aprobación de sus leyes, es decir, el Éxito Legislativo es mayor en los proyecto que son iniciados por la Presidencia $^{15}$.

\footnotetext{
${ }^{15}$ De acuerdo con García (2006, p. 81) esto puede ser explicado por el hecho de que "los Gobiernos se limitan a enviar proyectos que realmente querrían ver convertidos en leyes, lo cual incrementa su tasa de éxito, los legisladores presentan más proyectos, sabiendo previamente que tienen escasas o nulas posibilidades de ser aprobados. Este hecho obedece a la dificultad que tiene para un legislador situar en la agenda legislativa una iniciativa de su autoría y conlleva que el éxito del Poder Legislativo sea muy inferior aunque ello no significa que el Congreso no influya ni participe en la legislación que finalmente es sancionada".
} 


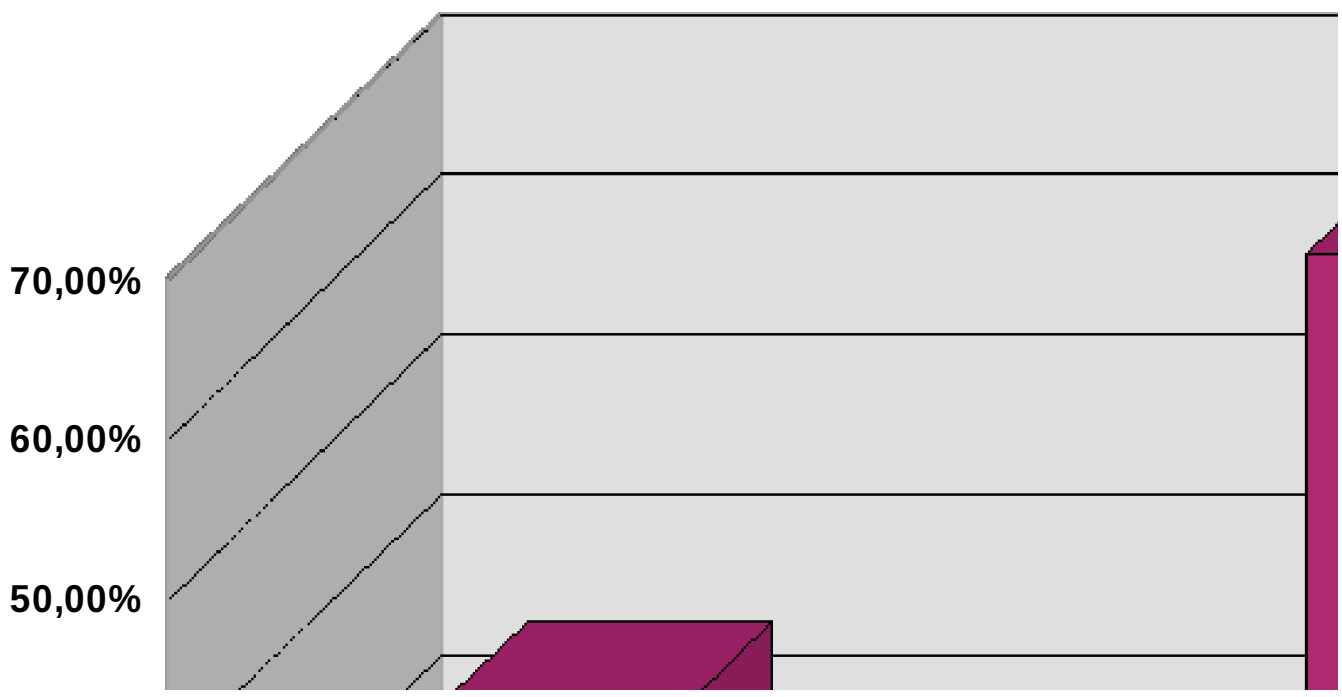

Fuente: Elaboración propia a partir de datos de las Cámaras de Diputados de Argentina y Brasil

Partiendo de la creación de un índice de Federalismo, elaborado a partir de las variables presentadas en la tabla abajo, fue posible hacer análisis estadísticas para verificar si hay relación entre el índice de federalismo y la dimensión eficacia del nivel de Gobernabilidad medidos a través del Éxito legislativo del ejecutivo. El resultado de este análisis indica que con el valor del Chi Cuadrado de Pearson $(0,157)$ se determina la existencia de relación significativa entre ambas variables, es decir, se elimina la hipótesis nula ( $\mathrm{HO})$ - no hay relación entre el grado de federalismo y el nivel de gobernabilidad - y se acepta la hipótesis alternativa $(\mathrm{H} 1)$ - hay relación entre el grado de federalismo y el nivel de gobernabilidad.

Tabla II: El Federalismo en Argentina y Brasil

\begin{tabular}{|c|c|c|}
\hline PAIS/ & & \\
VARIABLES & ARGENTINA & BRASIL \\
\hline
\end{tabular}


v. 8 - n. 2- agosto-dezembro/2011 - ISSN: 1806-5023

\begin{tabular}{||c|c|c||}
\hline PARLAMENTOS Y CONGRESO & $\begin{array}{c}\text { Bicameralismo } \\
\text { fuerte[4] }\end{array}$ & Bicameralismo fuerte[4] \\
\hline CONSTITUCIÓN & $\begin{array}{c}\text { Mayoría de dos } \\
\text { tercios[3] }\end{array}$ & Mayoría de un tercio[2] \\
\hline CONSTITUCIONALIDAD & $\begin{array}{c}\text { Constitucionalidad } \\
\text { Semifuerte[3] }\end{array}$ & $\begin{array}{c}\text { Constitucionalidad } \\
\text { Fuerte[4] }\end{array}$ \\
\hline BANCO CENTRAL & $\begin{array}{c}\text { Banco Central con } \\
\text { Independencia Plena[4] }\end{array}$ & $\begin{array}{c}\text { Banco Central con } \\
\text { Independencia Débil[2] }\end{array}$ \\
SOBREREPRESENTACIÓN & $\begin{array}{c}\text { Demos Constraining } \\
\text { Fuerte[4] }\end{array}$ & $\begin{array}{c}\text { Demos Constraining } \\
\text { Fuerte[4] }\end{array}$ \\
\hline
\end{tabular}

Fuente: Elaboración propia.

Así, al contrario de lo que afirma la literatura especializada, el nivel de gobernabilidad en los casos observados aumenta a medida que aumenta el grado de federalismo. Es decir, Argentina que marca 3,6 en una escala de federalismo que varia del 0 al 4 tiene $65,69 \%$ de éxito legislativo del Ejecutivo. En la misma línea, Brasil que tiene 3,2 en la escala de federalismo marca $37,96 \%$ en los niveles de éxito del Ejecutivo.

Gráfico II: Índice de Federalismo en Argentina y Brasil

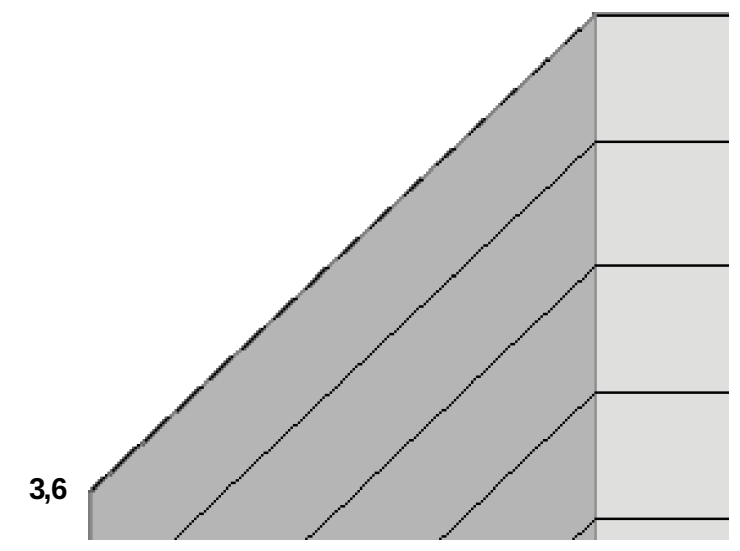

Fuente: elaboración Propia. 
Gráfico III: Porcentaje de Gobernabilidad Argentina y Brasil

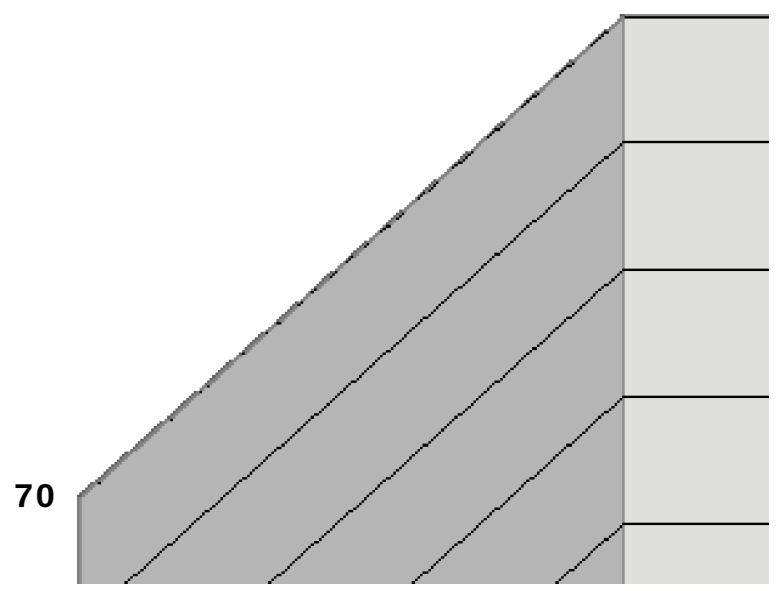

Fuente: elaboración Propia.

\section{CONSIDERACIONES FINALES}

Como ya se afirmaba en la introducción de este trabajo, en una realidad político-social caracterizada por la pluralidad es fundamental el intento de consensuar las diferentes opciones y voluntades. La búsqueda por un modelo de democracia consensual como promotora de una sociedad más igualitaria en detrimento de una "dictadura de la mayoría" es lo que propone Lijphart (2007) en sus análisis y es la opción que defiende este trabajo.

Con los dados presentados acerca de Argentina y Brasil para formar el índice de federalismo y el nivel de gobernabilidad demuestran una señal de que una democracia consensual, representada aquí por su vertiente federalista, no es incompatible con la gobernabilidad, todo lo contrario.

En los dos países analizados el nivel de gobernabilidad crece a medida que crece el índice de federalismo ${ }^{16}$. Así, se puede concluir

${ }_{16}$ Es importante reconocer que el modelo representado aquí no es, desde la perspectiva estadística, plenamente confiable, ya que el numero de casos es 
que en un nuevo escenario global, marcado por la diversidad, el Federalismo, entendido como un modelo de democracia consensual, es compatible con el buen gobierno, es decir, con la gobernabilidad en la arena política.

Como fue presentado a lo largo del texto, el análisis que se realiza en este trabajo se centra en una pequeña parte de la gobernabilidad, lo que nos impide de generar unas conclusiones más robustas sobre la relación entre gobernabilidades y federalismo. Sin embargo, con este trabajo se abre una amplia agenda de investigación. Por un lado, se puede ampliar el análisis de la gobernabilidad en los países estudiados a la hora de analizar la relación entre esta y la estructura federal, y por otro, existe la posibilidad de agregar otras indicadores a la medición del Federalismo propuesto por Lijphart, y usado en este trabajo. Además, se debe ampliar el numero de casos en el análisis, lo que generaría un análisis estadístico más sólida. Por lo tanto, se puede tomar diversos caminos para seguir analizando estos temas presentados aquí de manera incipiente e introductoria.

\section{REFERENCIAS}

ALCANTARA, Manuel. Gobernabilidad, Crisis y Cambio. México: Fondo de Cultura Económica, 1995.

ANASTASIA, Fátima et al. "Desde entonces hasta ahora: las condiciones y las instituciones de la democracia después de 1988", in Alcántara, Manuel e Ranulfo, Carlos (eds.). La Democracia Brasileña. Salamanca: Ed. Universidad de Salamanca, 2008.

ARBÓS, Xavier y GINER, Salvador. La gobernabilidad. Ciudadanía y democracia en la encrucijada mundial. Madrid: Editorial Siglo XXI, 1996.

demasiado pequeño. Sin embargo, lo que se propone este articulo es testear el modelo propuesto $y$, en una otra oportunidad, aplicarlo para un $n$ grande y obtener resultados estadísticos efectivamente validos. 
CAMARA DE DIPUTADOS DE ARGENTINA, http://www.diputados.gov.ar

CAMARA DE DIPUTADOS DE BRASIL, http://www.camara.gov.br

CONSTITUCIÓN DE ARGENTINA, Base de Datos Políticos de América Latina, http://pdba.georgetown.edu/

CONSTITUCIÓN DE BRASIL, Base de Datos Políticos de América Latina, http://pdba.georgetown.edu/

CUKIERMAN, Alex et al. Measuring Central Bank Independence and Its Effect on Policy Outcomes. San Francisco: ICS Press, 1992.

FERREIRA, Helder. A mensuração do grau de independência do Banco Central: uma análise de suas fragilidades, Revista de Economia Politica, São Paulo, outubro-dezembro/2006, vol.26, n.4 (104), p. 552563.

FRANCO, Rolando. Estado, consolidación democrática y gobernabilidad en América Latina. Revista América Latina Hoy. Salamanca: Instituto de Estudios de Iberoamérica y Portugal, Universidad de Salamanca, n. 8, 1994.

GARCÍA, Mercedes. Presidentes y Parlamentos: un análisis sobre el control de la actividad legislativa en América Latina, Tese de doutorado em Ciência Política, Programa de Doctorado Procesos Políticos Contemporáneos, Universidad de Salamanca. Salamanca, 2006.

GRIFFITHS, Ann y NERENBERG, Karl. Handbook of Federal Countries. Kingstom, Ontario: Queen's University, 2002.

HEREDIA VARGAS, Raimundo. Gobernabilidad: una aproximación teorica. Anales del IV Congreso Internacinal del CLAD sobre reforma del Estado y administración pública. Lisboa, 8-11 de octubre 2002.

HICKS, R. Globalization and Central Bank Independence: A partisan explanation. Princeton University, 2003. Disponível en $<\underline{\text { https://www.princeton.edu/ pcglobal/research/papers/hicks centralbank reform } 060}$ 3.pdf 
HOLANDA, Marcos y FREIRE, Leonardo. Medindo a independência do Banco Central Brasileiro. Texto para Discussão n. 228, Universidade Federal do Ceará, Pós-Graduação em Economia, Fortaleza, 2002.

HICKS, Raymond. Globalization and Central Bank Independence: A partisan explanation, en: http://www.princeton.edu/ pcglobal/research/papers/

LERNER, Emilia Raquel. El federalismo argentino. Revista América Latina Hoy. Salamanca: Universidad de Salamanca, vol. 6, 1993, p. 28-32.

LIJPHART, Arendt. Parliamentary versus Presidential Government. Oxford: Oxford University Press, 1992.

LIJPHART, Arendt. Modelos de democracia. Barcelona: Ariel, 2007.

LINZ, Juan. "Democracia presidencial o parlamentaria. ¿Hay alguna diferencia?", in: Linz, Juan. Presidencialismo vs. Parlamentarismo: Materiales para el estudio de la Reforma Constitucional. Buenos Aires: Concejo, Eudeba, 1988.

MELO, Marcos. "Hiperconstitucionalización y calidad de la democracia: mito y realidad", in: Alcántara, Manuel e Ranulfo, Carlos (eds.). La Democracia Brasileña. Salamanca: Ed. Universidad de Salamanca, 2008.

MUNK, Gerardo L. Gobernabilidad democrática a comienzos del siglo XXI: una perspectiva latinoamericana, Revista Mexicana de Sociología. México DF, año 65, n. 3, julio-septiembre 2003.

NOVESÀ, Marc Bou. El federalismo argentino: aproximación teórica y desempeño institucional. Barcelona: Documento de Trabajo n. 10, Institut Internacional de Governabilitat de Catalunya, 2005.

PACHANO, Simón. "Partidos y representación en la región andina", en: Kornblith, Miriam et al., Partidos Políticos en la Región Andina: entre la crisis y el cambio. Stockholm: IDEA, 2004.

REGIS, André. O Novo Federalismo Brasileiro. Rio de Janeiro: Companhia Editora Forense, 2009. 
REYNOSO, Diego. La diversidad institucional del bicameralismo en América Latina. Perfiles Latinoamericanos. Buenos Aires: Flacso, n. 35, enero-junio 2010, p. 105-143.

STEPAN, A. "Toward a New Comparative Politics of Federalism, (Multi)Nationalism, and Democracy: Beyond Rikerian Federalism", en: Stepan A. Arguing Comparative Politics. Oxford: Oxford University Press, 2001.

VALLĖS, J. M. Ciencia Política. Una introducción. Barcelona: Ed. Ariel, 2007.

WATTS, Robert. Comparing Federal Systems in 1990s, Ontario: Queen's University, 1996. 


\section{ANEXO}

\section{Datos Estadísticos:}

Tabla de contingencia Índice federalismo * Gobernabilidad * País

Recuento

\begin{tabular}{|l|l|l|r|r|r|}
\hline País & \multicolumn{4}{|c|}{} & \multicolumn{2}{|c|}{$\begin{array}{c}\text { dobernabilida } \\
\text { d }\end{array}$} & Total \\
\hline & \multicolumn{3}{|l|}{$\begin{array}{c}37,9 \\
6\end{array}$} & 65,69 & 37,96 \\
\hline $\begin{array}{l}\text { Argenti } \\
\text { na }\end{array}$ & $\begin{array}{l}\text { Indice } \\
\text { Federalismo }\end{array}$ & 3,6 & & 1 & 1 \\
\hline & Total & & 1 & 1 \\
\hline Brasil & $\begin{array}{l}\text { Indice } \\
\text { Federalismo }\end{array}$ & 3,20 & 1 & & 1 \\
\hline & Total & 1 & & 1 \\
\hline
\end{tabular}

Pruebas de chi-cuadrado

\begin{tabular}{|l|r|r|r|r|r|}
\hline & Valor & gl & $\begin{array}{r}\text { Sig. } \\
\text { asintótica } \\
\text { (bilateral) }\end{array}$ & $\begin{array}{c}\text { Sig. } \\
\text { exacta } \\
\text { (bilatera } \\
\text { l) }\end{array}$ & $\begin{array}{c}\text { Sig. } \\
\text { exacta } \\
\text { (unilatera } \\
\text { l) }\end{array}$ \\
\hline $\begin{array}{l}\text { Chi-cuadrado de } \\
\text { Pearson }\end{array}$ & $\begin{array}{r}2,000( \\
\text { b) }\end{array}$ & 1 &, 157 & & \\
\hline $\begin{array}{l}\text { Corrección por } \\
\text { continuidad(a) }\end{array}$ &, 000 & 1 & 1,000 & & \\
\hline $\begin{array}{l}\text { Razón de } \\
\text { verosimilitudes }\end{array}$ & 2,773 & 1 &, 096 & & \\
\hline $\begin{array}{l}\text { Estadístico exacto } \\
\text { de Fisher }\end{array}$ & 1,000 & 1 &, 317 & & \\
\hline $\begin{array}{l}\text { Asociación lineal } \\
\text { por lineal }\end{array}$ & 1,000 & \\
\hline
\end{tabular}




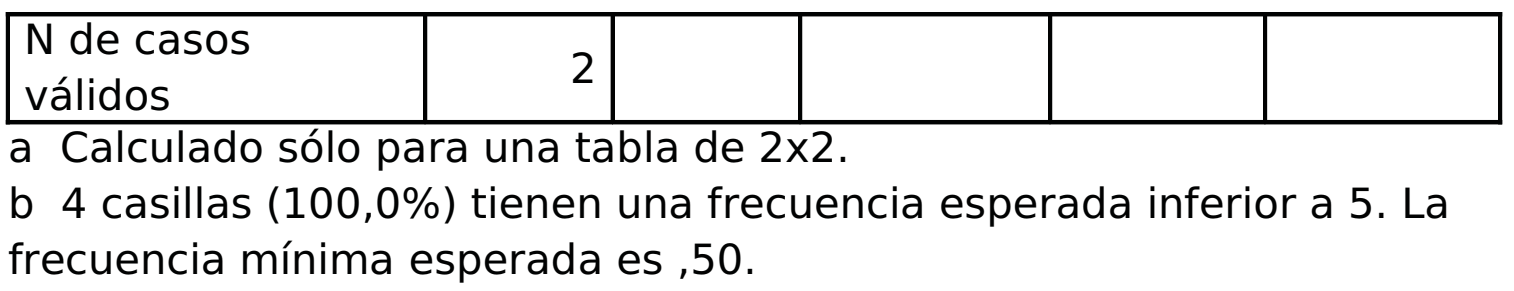

\section{Escalas de las Variables:}
A. Parlamentos y Congreso
[1] Bicameralismo débil
[2] Bicameralismo semifuerte I
[3] Bicameralismo semifuerte II
[4] Bicameralismo fuerte

\section{B. Constitución}

\section{[1] Mayorías ordinaria}

[2] Mayorías entre ordinarias y de dos tercios

[3] Mayorías de dos tercios o equivalentes

[4] Mayorías superiores a dos tercios

\section{Constitucionalidades}

[1] Sin revisión judicial

[2] Revisión judicial débil

[3] Revisión judicial semifuerte

[4] Revisión judicial fuerte

D. Banco Central

[1] Banco Central Dependiente

[2] Banco Central con Independencia Débil

[3] Banco Central con Independencia Fuerte

[4] Banco Central con Independencia Plena

E. Grado de de Sobrerepresentación en las Cámaras Territoriales

[1] Demos Enaibling Débil

[2] Demos Enaibling Fuerte

[3] Demos Constraining Débil

[4] Demos Constraining Fuerte 


\section{RESUMO}

El federalismo y la gobernabilidad no son variables opuestas, es decir, es posible conyugar en un mismo escenario el pluralismo político y la capacidad de actuación de los gobierno. Este articulo analiza la relación entre el grado de federalismo y la gobernabilidad, en concreto la capacidad del ejecutivo de aprobar sus leyes en el parlamento. Para tanto, se hará un análisis comparativo entre Argentina y Brasil ubicando estos países en una escala de federalismo a partir de algunas variables propuestas por Lijphart (2007) y Stepan (2001) en sus trabajos. El objetivo principal es saber si la ubicación de los países en esta escala tiene relación con la gobernabilidad en los términos presentados aquí.

PALABRAS CLAVES: federalismo, gobernabilidad, Brasil, Argentina.

\section{ABSTRACT}

Federalism and governability are not opposite variables, in other words, it is possible combine on the same stage the political pluralism and the capacity for action by government. This paper analyzes the relationship between the degree of federalism and the governability, specifically the executive's ability to approve laws in the parliament. For that, there will be a comparative analysis between Argentina and Brazil, locating these countries on a scale of federalism based in few variables proposed by Lijphart (2007) and Stepan (2001) in their works. So, the aim of this paper is understand the relation between the location of these countries on this scale and the governability in the terms presented here.

KEYWORDS: federalism, governability, Brazil, Argentina. 